\section{Nuances in diagnosis and management of acute esophageal necrosis}

\section{Grigoriy E. Gurvits}

New York University School of Medicine/Langone Medical Center, NY, USA

Dr. Dias et al present a comprehensive review on black esophagus (BE) [1], a rare but important entity in gastroenterology. The clinical significance of $\mathrm{BE}$, with its striking endoscopic appearance of necrotic mucosa preferably affecting the distal esophagus with a sharp transition at the gastrointestinal junction, has notably risen over the last decade, with advances in gastrointestinal endoscopy and its increased recognition in the medical literature [2]. It is therefore important to acknowledge Dr. Dias' work while commenting on some of the important aspects of acute esophageal necrosis (AEN) mentioned therein.

First, the role of vasoconstrictive agents for achieving mean arterial blood pressure control in sepsis may come at the expense of splanchnic blood flow, possibly resulting in a decrease in distal esophageal tissue perfusion. It is the secondary effect of such agents-namely, maintaining vital organ circulation in sepsis, an underlying critical condition-that may lead to decreased mortality in AEN. An interesting correlate would be to evaluate the potential delay in the time to esophageal mucosal healing and the risk of stricture formation in patients who have received vasoconstrictive agents. This could certainly be addressed in future studies.

Second, there have been a number of case reports linking the use of various drugs to the development of BE. While it is well known that the vasoconstrictive effects of cocaine produce a decrease in tissue perfusion, the causative relationship between some prescription medications and AEN described in the literature so far was often purely observational. The lack of scientific proof makes such a hypothesis likely to be coincidental. For example, bisphosphonates are known to cause a chemical injury-type tissue ulceration in the mid esophagus, typically due to the known anatomic impression from the aortic arch-an entity called "pill esophagitis"-but would not cause diffuse circumferential black-appearing tissue necrosis spanning the entire esophagus. Such reports of AEN are likely to be related to underlying or transient hemodynamic compromise in a vasculopathic patient and massive reflux of gastric contents in the setting of associated duodenal ulcer disease [3].

Third, management of AEN is aimed at correcting underlying medical conditions, hemodynamic support, nil-per-os restriction, and administration of high-dose proton pump inhibitor therapy. Surgical intervention is reserved for cases of esophageal perforation with mediastinitis and abscess formation [4]. Similar to left-sided ischemic colitis, AEN seems to correct on its own. Red blood cell transfusion may be indicated to correct gastrointestinal blood loss, but endoscopic therapy with submucosal injection of a racemic mixture of epinephrine seems dubious and potentially detrimental. Local tissue epinephrine therapy is a good tool in our endoscopic armament for direct lesional hemostasis, through its tamponade effect and vasoconstriction, precisely what one would want to avoid in ischemic organ injury. The risk of perforation is also highest in the esophagus, which notably lacks serosa, an additional layer of protection common to the rest of the gastrointestinal tract. It is for this reason that epinephrine injection should be avoided and, importantly, stent placement should not be performed. Indeed, there have been reports of perforated BE in the setting of stent use [5].

Finally, in asymptomatic patients, repeat endoscopy may be helpful in verifying the normalization of the mucosal lining and excluding occult pathology that could have been masked by diffuse BE on initial presentation. This may be performed a few weeks past therapy and should not delay the patient's discharge from the hospital once stable. Stricture or stenosis formation in AEN, which occurs in over $10 \%$ of cases during Stage 2 and 3 of the disease, may have an association with concurrent duodenal pathology [2] and seems to be inversely related to the state of immune compromise in affected patients with diabetes mellitus, malnutrition and malignancy [6]. Stricture or stenosis could be managed with outpatient endoscopic dilatation and antacid therapy, but repeat sessions may be necessary.

In the past decade, AEN has continued to ascend the differential diagnosis ladder in hospitalized patients presenting with upper gastrointestinal hemorrhage, largely because of the increased use of endoscopic procedures. Its prompt recognition and proper management will remain important for decreasing mortality and improving the patient's outcome.

\section{References}

1. Dias E, Santos-Antunes J, Macedo G. Diagnosis and management of acute esophageal necrosis. Ann Gastroenterol 2019;32:529-540.

2. Gurvits GE, Cherian K, Shami MN, et al. Black esophagus: new insights and multicenter international experience in 2014. Dig Dis Sci 2015; 60:444-453.

3. Kimura Y, Seno H, Yamashita Y. A case of acute necrotizing esophagitis. Gastrointest Endosc 2014;80:525-526.

4. Gurvits GE. Black esophagus: acute esophageal necrosis syndrome. World J Gastroenterol 2010;16:3219-3225.

5. Tse A, Basu S, Ali H, Hamouda A. Black necrotic oesophagus following the use of biodegradable stent for benign oesophageal stricture. J Surg Case Rep 2015;2015.

6. Gurvits GE, Shapsis A, Lau N, Gualtieri N, Robilotti JG. Acute esophageal necrosis: a rare syndrome. J Gastroenterol 2007;42:29-38.

Department of Medicine, Division of Gastroenterology, New York University School of Medicine/Langone Medical Center, NY, USA

Conflict of Interest: None

Correspondence to: Grigoriy E. Gurvits, MD, FACP, FACG, AGAF, Clinical Professor, Department of Medicine, Division of Gastroenterology, New York University School of Medicine/Langone Medical Center, 555 Madison Avenue, $3^{\text {rd }}$ Floor, New York, NY 10022, USA, e-mail: g_gurvits@hotmail.com

Received 20 February 2020; accepted 9 March 2020; published online 10 May 2020

DOI: https://doi.org/10.20524/aog.2020.0488 